\title{
A importância da Odontologia do Esporte no rendimento do atleta
}

\author{
The importance of Sports Dentistry in the athlete's performance \\ La importancia de la Odontología Deportiva en el rendimiento del deportista
}

Recebido: 09/03/2021 | Revisado: 14/03/2021 | Aceito: 17/03/2021 | Publicado: 24/03/2021

\author{
Kevin Gabriel Teixeira \\ ORCID: https://orcid.org/0000-0003-1247-7335 \\ Faculdade Sant'Ana, Brasil \\ E-mail: kevinteixeiraedf@gmail.com \\ Alessandro Bodanese \\ ORCID: https://orcid.org/0000-0002-5224-2664 \\ Faculdade Sant'Ana, Brasil \\ E-mail: alebodanese97@gmail.com \\ João Karllos Pinho Bandeira \\ ORCID: https://orcid.org/0000-0002-7994-8296 \\ Faculdade Paulo Picanço, Brasil \\ E-mail: joaokarllos@yahoo.com.br \\ Márcia Rezende \\ ORCID: https://orcid.org/0000-0001-8474-5656 \\ Faculdade Paulo Picanço, Brasil \\ Faculdade Sant'Ana, Brasil \\ E-mail: rezendemarcia@outlook.com
}

\begin{abstract}
Resumo
O objetivo do presente estudo foi compreender a importância da Odontologia do Esporte para a saúde do atleta, verificar as doenças bucais mais prevalentes em atletas de alto rendimento e identificar a influência de doenças bucais no rendimento do atleta. Foram realizadas buscas na literatura, nas seguintes bases de dados eletrônicas: Biblioteca Virtual em Saúde, SciELO, Google Acadêmico, Pubmed e Portal de Periódicos Capes/MEC. Observou-se que os cuidados bucais vão além da indicação do uso de protetores bucais. O cirurgião-dentista atua na prevenção e intervenção de fatores prejudiciais como a doença cárie, disfunção temporomandibular, doença periodontal, má oclusão, hábitos deletérios, erosão dental, além de traumatismos dentários e orofaciais causados pelo impacto em esportes de contato. A atenção se dá inclusive na prescrição de medicamentos, para que não corra o risco de resultado positivo no controle de doping. Uma das preocupações em tratar as doenças bucais, se dá pelo fato das repercussões sistêmicas, pois podem prejudicar a capacidade de regeneração muscular, gerar problemas respiratórios e complicações cardiovasculares. Além de comprometer a eficiência mastigatória, a fala, favorecer traumas dentários, e também diminuir a disposição e capacidade de concentração dos atletas nos casos de dor. Há necessidade da realização de mais estudos sobre o tema e da divulgação no meio esportivo da importância da saúde bucal para a manutenção da saúde sistêmica e, consequente, melhoria do desempenho esportivo dos atletas.
\end{abstract}

Palavras-chave: Odontologia; Esportes; Exercício físico; Lesões do esporte; Patologia bucal; Traumatismos dentários.

\begin{abstract}
The aim of the present study was to understand the importance of Sports Dentistry for the athlete's health, to verify the most prevalent oral diseases in high-performance athletes and to identify the influence of oral diseases on the athlete's performance. Literature searches were carried out in the following electronic databases: Virtual Health Library, SciELO, Google Scholar, Pubmed and Portal de Periódicos Capes / MEC. It was observed that oral care goes beyond the indication of the use of mouthguards. The dentist acts in the prevention and intervention of harmful factors such as caries disease, temporomandibular disorder, periodontal disease, malocclusion, deleterious habits, dental erosion, in addition to dental and orofacial trauma caused by the impact on contact sports. Attention is even given to the prescription of medications, so that there is no risk of a positive result in doping control. One of the concerns in treating oral diseases, is due to the fact of the systemic repercussions, as they can impair the capacity of muscle regeneration, generate respiratory problems and cardiovascular complications. In addition to compromising masticatory efficiency, speech, favoring dental trauma, and also decreasing athletes' willingness and ability to concentrate in cases of pain. There is a need for further studies on the subject and for the dissemination in the sports environment of the importance of oral health for the maintenance of systemic health and, consequently, improvement of the athletes' sports performance.
\end{abstract}

Keywords: Dentistry; Sports; Exercise; Athletic injuries; Pathology oral; Tooth injuries. 


\section{Resumen}

El objetivo del presente estudio fue comprender la importancia de la Odontología Deportiva para la salud del deportista, verificar las enfermedades bucodentales más prevalentes en deportistas de alto rendimiento e identificar la influencia de las enfermedades bucodentales en el rendimiento del deportista. Se realizaron búsquedas bibliográficas en las siguientes bases de datos electrónicas: Biblioteca Virtual en Salud, SciELO, Google Scholar, Pubmed y Portal de Periódicos Capes / MEC. Se observó que el cuidado bucal va más allá de la indicación del uso de protectores bucales. El cirujano dentista actúa en la prevención e intervención de factores nocivos como la enfermedad de caries, trastorno temporomandibular, enfermedad periodontal, maloclusión, hábitos deletéreos, erosión dental, además de los traumatismos dentales y orofaciales provocados por el impacto en los deportes de contacto. Incluso se presta atención a la prescripción de medicamentos, para que no exista riesgo de un resultado positivo en el control de dopaje. Una de las preocupaciones en el tratamiento de las enfermedades bucodentales, se debe a las repercusiones sistémicas, ya que pueden perjudicar la capacidad de regeneración muscular, generar problemas respiratorios y complicaciones cardiovasculares. Además de comprometer la eficiencia masticatoria, el habla, favorece el trauma dental y también disminuye la disposición y capacidad de concentración de los deportistas en casos de dolor. Se necesitan más estudios sobre el tema y la difusión en el ámbito deportivo de la importancia de la salud bucal para el mantenimiento de la salud sistémica y, en consecuencia, la mejora del rendimiento deportivo de los deportistas.

Palabras clave: Odontología; Deportes; Ejercicio físico; Lesiones en deportes; Patología bucal; Traumatismos de los dientes.

\section{Introdução}

Para um bom rendimento do atleta, o corpo em sua totalidade, precisa estar saudável, sendo importante o acompanhamento por uma equipe multidisciplinar. Um dos profissionais que integram a equipe é o cirurgião-dentista, especialista em Odontologia do Esporte, devido ao impacto que a saúde bucal gera na saúde geral do atleta, refletindo no seu desempenho (Padilha \& Namba, 2016).

A Odontologia do Esporte foi reconhecida como uma nova especialidade na Odontologia brasileira em 2015 (CFO, Acesso em: 24 de jul. 2020), a qual não se resume apenas a indicação do uso de protetores bucais como medida de prevenção, mas também a orientação para manutenção da saúde bucal e o tratamento de doenças que acometem a cavidade bucal (Costa, 2009).

A Odontologia do Esporte promove a saúde bucal prevenindo e intervindo em fatores prejudiciais como a doença cárie, Desordens na Articulação Temporomandibular (DTM), má oclusão, traumas dentários e orofaciais, Doença Periodontal (DP), entre outros. Em consequência, pode ocorrer à restrição alimentar, dor, prejudicar o repouso, reduzir o desempenho nos treinamentos ou até mesmo o afastamento do atleta de competições (Bastos et al., 2013).

Alguns cuidados odontológicos passaram a ser considerados pelas equipes técnicas, pelo fato de estarem diretamente relacionados ao rendimento e a qualidade de vida, e podem ser cruciais para a vitória em competições e/ou quebra de recordes (Alves et al., 2017).

A prevalência de doenças bucais em atletas ainda é um fator preocupante, e por isso uma atenção especial tem sido destinada a prevenção e tratamento dessas alterações (Needleman et al., 2013). O processo inflamatório destrutivo desencadeado por bactérias, além de acometerem os dentes e seus tecidos de proteção e suporte (gengiva e osso), podem gerar complicações como aterosclerose, infecções respiratórias e o infarto do miocárdio (Bastos et al., 2013). Além disso, o proces so inflamatório crônico pode afetar o sistema muscular, dificultando a recuperação tecidual (Souza et al., 2016). Tais fatores podem impactar na qualidade de vida dos atletas, provocar queda de rendimento e alterar a performance (Costa, 2009).

Assim sendo, o objetivo do presente estudo foi compreender a importância da Odontologia do Esporte para a saúde do atleta, verificar as doenças bucais mais prevalentes em atletas de alto rendimento e identificar a influência de doenças bucais no rendimento do atleta. 


\section{Metodologia}

O presente estudo se caracteriza como uma pesquisa bibliográfica em função dos procedimentos técnicos e em exploratória de acordo com seus objetivos (Gil, 2010). Trata-se de uma revisão narrativa da literatura, que são publicações amplas, para descrever o "estado da arte" sobre um determinado assunto, consistindo da análise da literatura publicada na interpretação e análise pessoal do autor. Apesar da impossibilidade de reprodução, as revisões narrativas tem um papel importante na educação continuada por contribuir com a aquisição e atualização do conhecimento em curto espaço de tempo (Rother, 2007).

Para a realização do presente estudo foram realizadas buscas na literatura em bases de dados eletrônicas, como Biblioteca Virtual em Saúde, SciElO, Google Acadêmico, Pubmed e Portal de Periódicos Capes/MEC, para obtenção de artigos científicos, dissertações e teses, e também através de livros, revistas e periódicos sobre o tema.

Para a busca dos artigos foram utilizados os descritores de busca: Odontologia, Esporte, traumatismos dentários, dopping, bruxismo, má oclusão, erosão dental, disfunção temporomandibular, cárie dental, patologia bucal, doença periodontal. Os termos foram combinados com auxílio dos operadores booleanos AND e OR.

Foram incluídos estudos publicados no período de 2000 a 2020, sem restrição de idioma e de tipo de estudo. Foram excluídos os estudos que não estivessem disponíveis na íntegra, artigos opinativos que não estivessem embasados em evidências científicas, e os estudos que apesar de abordar temas da Odontologia, não estivessem relacionados a atletas ou a Odontologia do Esporte.

Após o levantamento bibliográfico, procedeu-se a seleção e análise das publicações. Inicialmente as publicações potencialmente elegíveis foram selecionadas através dos títulos e resumos. Os artigos completos foram obtidos e selecionados de acordo com os critérios de inclusão e exclusão.

\section{Revisão de Literatura e Discussão}

\subsection{Odontologia do Esporte}

A Odontologia do Esporte teve início em 1890, no Reino Unido, através do trabalho do cirurgião-dentista Woolf Krause, que criou um dispositivo de guta-percha, para proteção dos dentes anteriores dos boxeadores. Em 1913, seu filho Phillp Krause fabricou um protetor bucal reutilizável, exclusivamente para o boxeador Ted "Kid" Lewis, também como a finalidade de proteção dos tecidos bucais durante o combate, mas com a vantagem de ser reutilizável (Andrade et al., 2017; Barberini et al., 2002). E em 1930, surgiram os primeiros relatos em revistas científicas, detalhando a confecção de protetores bucais feitos de borracha vulcanizada para lutadores de boxe (Gialain, 2015; Jacobs, 1930).

A Odontologia do Esporte surgiu devido a necessidade de especializar a Odontologia para o Esporte, sendo uma das disciplinas de grande importância na composição da equipe multidisciplinar (Padilha \& Namba, 2016). Tem como foco o estudo, revisão, prevenção e tratamento da traumatologia orofacial, a manutenção da saúde bucal do atleta e a difusão de informações e novos conhecimentos na comunidade esportiva (Gay-Escoda et al., 2011), e não meramente curativa em momentos pós-lesão (Lima et al., 2019).

O cirurgião-dentista passou a fazer parte da equipe multidisciplinar de clubes e participar de grandes eventos esportivos, como as Olimpíadas, acompanhando a equipe e dando suporte aos atletas (Needleman et al., 2013).

A importância da prevenção no esporte ficou evidente após relatório da Copa do Mundo de 2010, pela Federação Internacional de Futebol, em que todas as equipes realizaram exames preventivos nos jogadores, refletindo durante os jogos em menores índices de lesão por partida comparado aos anos anteriores (Padilha \& Namba, 2016).

O primeiro trabalho relacionado a Odontologia do Esporte no Brasil foi durante a Copa do Mundo de Futebol na Suécia, no ano de 1958, efetuado pelo cirurgião-dentista da Seleção Brasileira de Futebol Dr. Mario Trigo. Devido a origem 
humilde, muitos jogadores apresentavam problemas dentários, o que impactava negativamente na performance. O Brasil foi o único país que levou um cirurgião-dentista e um psicólogo nesta Copa, demonstrando a preocupação em proporcionar uma estrutura completa para seus atletas. Trigo também acompanhou a equipe em 1962, 1966 e 1970 (Padilha \& Namba, 2016).

Em seu livro "O Eterno Futebol", Trigo relatou que o maior atraso na recuperação de lesões em atletas, eram os que apresentavam problemas dentários, o que o levou a investigar a relação entre a existência de focos dentários infecciosos e a demora na recuperação de lesões. Observou após tratamento, que a recuperação ocorria com maior rapidez (Amorim \& Añez, 2017; Padilha \& Namba, 2016; Trigo, 2002). Em virtude do ocorrido, a Confederação Brasileira de Desportos, conhecia atualmente como Confederação Brasileira de Futebol, solicitou que Trigo realiza-se um planejamento odontológico para a seleção brasileira de futebol. Após avaliação dos 33 jogadores, Trigo realizou 118 extrações e no exame radiográfico haviam muitos focos infecciosos. Relatou que o trabalho odontológico aliado ao trabalho da equipe de saúde, melhorou o desempenho dos jogadores em campo (Amorim \& Añez, 2017; Trigo, 2002).

Após a saída de Trigo, Carlos Sergio Augusto assumiu os cuidados da saúde bucal dos jogadores, representando a Seleção Brasileira de Futebol em 1992, 1994,1998 e 2002. Realizou um convênio com a Associação Fluminense de Educação, para diagnóstico bucal, atendimentos de urgência e ações preventivas, para as seleções masculina e feminina desde a base até profissional (Padilha \& Namba, 2016).

A Odontologia do Esporte vem crescendo e alguns clubes como Atlético Mineiro, Botafogo, Corinthians, Flamengo, São Paulo, Ceará e Coritiba, possuem um setor específico para a saúde bucal dentro de seus clubes ou centro de treinamento. O que favorece a adesão ao tratamento odontológico por parte dos atletas, principalmente os de alto rendimento, evitando que em seus escassos momentos de folga precisem buscar tratamento (Padilha \& Namba, 2016).

Os cirurgiões-dentistas desses clubes de futebol trabalham de maneira preventiva, através de palestras de conscientização, orientação sobre higienização bucal e da influência da dieta no risco de cárie dental e na ação do desgaste dental. Além disso, os esportistas são apresentados sobre os riscos de traumatismos e da importância de utilizar medicamentos apenas com prescrição, pois alguns medicamentos utilizados para doenças bucais podem acarretar doping. Também são realizados exames clínicos e radiográficos para diagnóstico e tratamento, ou apenas para controle de tratamento executados anteriormente, visando a saúde e o melhor desempenho nas atividades esportivas, evitando traumas e acidentes (Antunez \& dos Reis, 2010; Assis, 2013).

\subsection{Principais problemas dentários que afetam os esportistas}

Apesar da prática esportiva estar relacionada a um estilo de vida saudável, as doenças bucais são encontradas frequentemente em atletas e podem ser responsáveis por impactar negativamente o bem-estar, o treinamento, o desempenho, bem como a saúde geral (Souza, 2017). O rendimento físico de um atleta pode ser reduzido em $21 \%$ se tiver algum distúrbio na cavidade bucal (Antunez \& dos Reis, 2010).

Ashley et al. (2015) realizaram uma revisão sistemática para determinar a epidemiologia de doenças bucais em atletas de elite e a associação com o desempenho desportivo. Observaram que a saúde bucal dos atletas é precária, em uma ampla variedade de esportes, podendo afetar o desempenho atlético. Sendo importante o desenvolvimento de pesquisas com amostras representativas, para avaliar a saúde bucal e a relação com o desempenho dos atletas. Dentre os problemas bucais mais encontrados estavam: cárie dentária, doença periodontal, má oclusão, DTM, erosão dental, traumatismos e terceiros molares impactados.

\subsubsection{Cárie Dentária}

A cárie dentária é uma doença infecciosa e multifatorial, sendo necessário para seu estabelecimento a intersecção de 
seus fatores determinantes: hospedeiro, microbiota e dieta, por um determinado período (Bonecker et al., 2012). Caracteriza-se por apresentar um desequilíbrio entre a perda e ganho de minerais dos tecidos dentários, decorrente do metabolismo bacteriano e que pode haver predominância da desmineralização, resultando em perda mineral e consequente possibilidade de cavitação (Batista et al., 2020).

Nos atletas, a cárie dentária tem sido predominante em comparação com as outras alterações bucais. Conforme verificado na avaliação bucal realizada durante os jogos Olímpicos de Londres, que mais da metade dos atletas apresentava cárie dentária $(55,1 \%)$, destas $41 \%$ eram irreversíveis (Needleman et al., 2013), bem como na revisão sistemática apresentada por Ashley et al. (2015) em que 75\% dos atletas apresentavam cárie dentária.

Muitos são os desafios para a saúde bucal dos atletas. A dieta, muitas vezes composta por géis e bebidas com valores elevados de carboidratos, um dos fatores causais para cárie. A perda de líquidos corporais pela intensa sudorese e grandes esforços físicos, pode contribuir com aumento da densidade, redução do fluxo e composição da saliva, e também com picos de ação bacteriana, similares aos que ocorrem no período do sono. Dessa forma, há redução dos efeitos remineralizantes da saliva e de sua atividade antimicrobiana, aumentando o impacto dos carboidratos na cárie dentária e da acidez das bebidas do tipo repositores, na erosão dental (Needleman et al., 2015; Pastore et al., 2017).

As cáries dentárias, se não tratadas progridem, causando dor e podem levar a necessidade de tratamento endodôntico (tratamento de canal) e perda do elemento dental. Essas enfermidades na cavidade bucal geram distúrbios da mastigação, dificultando a alimentação e consequentemente um menor aproveitamento energético, comprometendo o desempenho físico e descanso do atleta (Antunez \& dos Reis, 2010).

Diante desse contexto, torna-se importante o controle mecânico do biofilme dental através da higienização bucal, orientações sobre hábitos alimentares e acompanhamento pelo cirurgião-dentista (Lima et al., 2019).

\subsubsection{Doença Periodontal}

A DP é uma doença multifatorial, que tem como principal característica a inflamação crônica dos tecidos de suporte. Está entre as patologias infecciosas mais prevalentes no mundo, afetando cerca de 20-50\% da população global. Alta prevalência de doença periodontal em adolescentes, adultos e idosos o torna uma preocupação de saúde pública (Nazir, 2017).

O biofilme dental, conhecido popularmente como placa bacteriana, contém microrganismos que provoca a inflamação da gengiva (gengivite), que se não tratada, evolui para a periodontite. A DP resulta da resposta inflamatória gerada pelas bactérias presentes no biofilme dental, que ativam o sistema imunológico do paciente determinando o resultado final da doença (Silveira \& Alves, 2009).

Ashley et al. (2015) verificaram que 76\% dos atletas apresentavam gengivite e 15\% possuíam DP moderada a grave. Uma das preocupações em manter a saúde gengival dos atletas e a cavidade bucal livre de infecções, se dá pelo fato das repercussões sistêmicas, já que os processos infecciosos da cavidade bucal podem disseminar para o restante do corpo (Padilha, 2015).

Estudos prévios indicaram relação entre DP e doenças cardiovasculares, em que bacteremias podem ocorrer de forma assintomática. Um terço dos infartos de miocárdio ocorre em indivíduos que não apresentam fatores de risco como: hipertensão arterial, hipercolesterolemia, diabetes e tabagismo. Ou seja, outros fatores de risco podem apresentar papel importante, como as infecções crônicas bucais (Abou-Raya et al., 2002).

Há evidências científicas comprovando a relação entre DP e lesões musculares. A DP é capaz de modificar células e mediadores inflamatórios, atuando indiretamente no catabolismo muscular (Dalri, 2020). Um dos marcadores inflamatórios que evidenciam essa conexão é o nível de creatina quinase, uma enzima de alto peso molecular, que é considerada sensível para o diagnóstico de lesão muscular. O nível sérico dessa enzima pode ser marcador funcional do tecido muscular, um 
aumento pode ser sinal de necrose celular ou lesão tecidual muscular (Souza \& Ribas, 2009). A DP gera altos níveis de outros marcadores como as citocinas inflamatórias circulantes que podem causar fadiga muscular, dificultar a recuperação de lesões musculares, comprometer a saúde geral e desempenho do atleta (Dalri, 2020; Souza \& Ribas, 2009).

Souza (2013) analisou a alteração do perfil inflamatório em ratos, causada pela relação entre DP e exercício físico intenso. Observou que os ratos com DP que realizaram exercícios físicos apresentaram fibras musculares de menor perímetro e maior quantidade de células inflamatórias. A DP influencia o processo metabólico que envolve a hipertrofia muscular, afetando tanto o sistema musculoesquelético quanto a recuperação tecidual.

Pacientes com DP apresentaram níveis séricos de proteína c-reativa e interleucina-6 elevados, quando comparados a pacientes sadios. Contudo, observou-se uma redução significante nos níveis destes marcadores inflamatórios após a realização do tratamento da DP (Nakajima et al., 2010).

A inflamação é importante para recuperação tecidual, tanto nos casos de microlesões que resultam no aumento de massa muscular, quanto nos casos de lesões advindas de traumas e estiramentos, que ocorre a regeneração tecidual (Souza et al., 2016), por isso é importante que não ocorra sobreposição de processos inflamatórios.

O uso de esteroides anabolizantes pode fazer com que o tecido gengival fique hiperplásico, devido a presença de receptores específicos para andrógenos nos fibroblastos gengivais, que se duplicam quando o tecido gengival está inflamado (Fronza, 2020).

\subsubsection{Má Oclusão}

A oclusão dental se estabelece através do contato dental entre os dentes da maxila e da mandíbula. O termo oclusão normal é utilizado para caracterizar a relação anatômica adequada entre os dentes e as demais estruturas do sistema estomatognático (Werutski \& Falchetti, 2016).

A má oclusão é um dos problemas bucais frequentes na população, pela alta prevalência é considerado um problema de saúde pública (Garib et al., 2013). Aproximadamente 70\% da população possui má oclusão, 27\% oclusão aceitável e apenas $3 \%$ possui oclusão ideal (Freitas, 2013).

Os desvios da normalidade originam as más oclusões, as quais podem ser dentárias, esqueléticas ou dentoesqueléticas (Santos-Coluchi \& Simplício, 2011). As más oclusões resultam da interação de fatores genéticos e ambientais (Garib et al., 2013).

Dentre os fatores genéticos estão o tipo facial, padrão esquelético sagital da face, anomalias dentárias e craniofaciais. As irregularidades genéticas não podem ser evitadas, mas podem ser atenuadas com tratamento ortopédico, ortodôntico e cirurgias ortognáticas (Garib et al., 2013).

Os fatores etiológicos ambientais são os não genéticos, relacionados ao meio em que se vive, como: traumatismos, perda precoce de dentes decíduos e/ou permanentes, e os hábitos bucais deletérios (Garib et al., 2013).

Um dos hábitos deletérios encontrados frequentemente nos atletas é o bruxismo. $\mathrm{O}$ bruxismo pode ser cêntrico caracterizado pelo apertamento dos dentes e excêntrico pelo ranger dos dentes. A força gerada pode promover danos como desgaste dental, doenças periodontais, problemas na Articulação Temporomandibular (ATM), hipersensibilidade, mobilidade dental, dores de cabeça e muscular. Trata-se de um problema multifatorial e pode ser desencadeado devido a ansiedade, estresse, problemas respiratórios e contatos prematuros entre os dentes. $\mathrm{O}$ tratamento deve ser realizado por uma equipe multidisciplinar, com a participação do cirurgião-dentista, médico otorrinolaringologista, fonoaudiólogo e psicólogo (Rodrigues, 2006; Soares et al., 2004).

O hábito do bruxismo é verificado em atletas, como por exemplo nos praticantes de Karate, que cerram os dentes antes de realizar um ataque. Esse comportamento também é verificado em corredores, jogadores de tênis e praticantes de 
crossfit. Durante o apertamento dental a musculatura da face e pescoço é contraída, e esse trabalho contínuo pode desencadear dores de cabeça, dores musculares e limitação para abertura de boca. Existem alguns protetores bucais que possuem um design diferente, que além da proteção contra trauma, promovem o relaxamento muscular e protegem a estrutura dental, favorecendo os atletas que possuem bruxismo (Namba, 2018; Raquel et al., 2016).

Outro hábito encontrado com frequência entre os atletas, é a respiração bucal, que pode ser causada por alergias, bloqueios das vias aéreas superiores, desvio do septo nasal, inflamação dos cornetos, adenoides hipertróficas, que agem bloqueando o acesso do ar na cavidade nasal. A respiração bucal modifica a postura da cabeça, garantindo uma abertura suficiente que possibilite a respiração (Garib et al., 2013). A face do respirador bucal é alongada, o olhar baixo, lábios entre abertos, hipotônicos e ressecados. Existe relação entre respiração bucal e fadiga, sono durante o dia, falta de apetite e falta de atenção (Bastos et al., 2013).

Respiração bucal em atletas, faz com que cansem mais rápido, comprometendo o rendimento físico. Na respiração nasal, o ar é aquecido, filtrado e umidificado, mas quando inalado através da boca, o ar entra em temperatura ambiente, com impurezas, e faz com que haja ressecamento da mucosa bucal favorecendo aparecimento de DP, infecções nas tonsilas palatinas, maior gasto de energia para a aspiração, atrapalhando o desempenho físico (Bastos et al., 2013).

Nos atletas, há um alto percentual de má oclusão, no estudo de Souza et al. (2011) observaram que 47\% dos atletas possuíam má oclusão, apresentando sobremordida profunda, mordida aberta, diastemas e apinhamento dental. Essas alterações podem interferir na respiração, deglutição, além de contribuir para a ocorrência de traumas dentários (Souza et al., 2011).

A má oclusão pode gerar problemas mastigatórios, dificultando a trituração dos alimentos e consequentemente interferindo na absorção de nutrientes, o que pode afetar no desempenho esportivo (Lima et al., 2019). Além disso, pode levar a problemas de fonação, perda de equilíbrio muscular, dor de cabeça, problemas na ATM e desconforto (Souza et al., 2011).

A má oclusão pode favorecer desequilíbrios posturais. Alguns autores defendem a hipótese de que as más oclusões levariam a uma compensação dos músculos do pescoço e ombros, interferindo na postura. Como por exemplo, os respiradores bucais por respirarem predominantemente pela boca, alteram a postura para facilitar a passagem de ar pela faringe, anteriorizando a cabeça e desencadeando um desequilíbrio muscular de todo eixo postural. Ocorre diminuição da atividade do diafragma e hipoatividade da musculatura abdominal. Essas alterações causam um progressivo enfraquecimento muscular pelo menor esforço respiratório (Saga et al., 2011), reduzindo o aproveitamento de oxigênio principalmente durante o exercício.

O tratamento das más oclusões pode ser realizado com aparelhos fixos ou removíveis, e nos casos de desarmonia esquelética entre maxila e mandíbula pode haver necessidade de tratamento ortodôntico-cirúrgico, através da realização de cirurgia ortognática (Nunes et al., 2020).

Os aparelhos fixos contribuem para uma maior gravidade nos traumas sobre os tecidos moles labiais ocorridos durante a prática esportiva. Os aparelhos instalados na face lingual, apesar de reduzir esse problema, podem causar lesões na língua e assoalho bucal. Os aparelhos fixos também podem dificultar a higienização, a alimentação e faz com que os protetores bucais se tornem muito volumosos, dificultando a adesão ao tratamento por parte dos atletas. Uma alternativa de tratamento seria a utilização dos alinhadores estéticos, que por serem removíveis facilitam a higienização, reduzem os danos aos tecidos bucais causados por traumas e viabilizam a confecção de protetores bucais menos volumosos, contudo requer disciplina quanto ao uso e os custos podem ser altos (Nunes et al., 2020).

Os aparelhos favorecem o acúmulo de alimentos e biofilme dental, sendo um fator de risco adicional para cárie dental e DP, nesses casos os cuidados com a higienização devem ser redobrados, para que não comprometa a saúde bucal e geral do atleta (Nunes et al., 2020). 


\subsubsection{Disfunção Temporomandibular}

As DTMs são alterações que envolvem a musculatura mastigatória e as ATMs. São as maiores causas das dores orofaciais, excluindo as dores dentárias, e causam ruídos articulares e limitação dos movimentos bucais. Possuem etiologia multifatorial, sendo causadas por alterações anatômicas, traumas, desequilíbrios oclusais, hábitos parafuncionais e estresse. As manifestações clínicas mais frequentes são dor, espasmo muscular, crepitação e disfunção articular (Bonotto \& Bonotto, 2016; Okeson \& de Leeuw, 2011).

Um dos fatores que mais contribui para o surgimento das DTMs são os traumas, o que é muito frequente nas práticas esportivas, principalmente nos esportes de contato (Weiler et al., 2010).

A prevalência de sinais e sintomas de DTMs foram avaliados em adolescentes do sexo masculino, sendo 46 jogadores 41 não atletas. Vinte porcento dos voluntários relataram pelo menos um sinal ou sintoma de DTM, não havendo diferença significativa entre atletas e não atletas. Dentre os sintomas, o mais prevalente foi dor muscular mastigatória à palpação (66,8\% atletas e $60 \%$ não atletas). O hábito de apertamento ou ranger os dentes foi relatado por 50\% dos atletas e $20 \%$ para não atletas, e dor de cabeça ocorreu em $25 \%$ dos atletas e $20 \%$ em não atletas. O fato de não haver diferença entre os grupos, pode ter sido em função da amostra ser pequena e composta por jovens, já que DTM grave é rara nessa faixa etária (Weiler et al., 2010). Além disso, pode ter ocorrido também uma ação protetora da testosterona, que pode atenuar os danos da ATM, enquanto o estrogênio pode exacerbar (Flake et al., 2006).

Bonotto (2013) avaliou a prevalência de DTM em atletas lutadores de alta performance, atletas recreativos e indivíduos não-atletas. Verificou maior prevalência de DTM em atletas profissionais quando comparado com os outros grupos. O deslocamento de disco articular foi o achado mais frequente, e a dor de baixa intensidade. Atletas recreativos apresentaram índices de DTM semelhantes aos não praticantes de artes marciais.

O tratamento conservador e reversíveis devem ser priorizados para o tratamento de DTM, como uso de placas miorrelaxantes e mudança de hábitos. Os atletas que usam protetor bucal devem fazer controles periódicos para checar a retenção e ajuste oclusal, pois protetores soltos podem estimular o apertamento dental e os desajustes oclusais podem sobrecarregar as ATMs (Bonotto \& Bonotto, 2016).

\subsubsection{Erosão Dental}

A erosão dental, também denominada biocorrosão, é caracterizada pela dissolução dos tecidos dentais devido a exposição constante a ácidos intrínsecos e extrínsecos, e enzimas proteolíticas. Ocorre em níveis de pH abaixo de 4,5 e dessa forma o processo de desmineralização sobrepõem a ação remineralizadora da saliva. Inicialmente ocorre a perda de esmalte e posteriormente de dentina, de forma crônica e irreversível. Com a alteração da microdureza, o esmalte dental fica mais susceptível a outras formas de desgaste dental como a abrasão e a atrição (Cury et al., 2017; Grippo et al., 2012; Lussi \& Jäggi, 2008).

Atletas que praticam esportes como corrida, natação, ciclismo e triatlo, exigem um treinamento intenso e em alta frequência, e por esse motivo estão mais sujeitos a desenvolver a erosão dental, por estarem mais expostos a um pH mais baixo, em virtude da hiposalivação (Frese et al., 2015; Leite, 2019).

Os atletas também estão expostos a ácidos extrínsecos, pela ingestão de bebidas isotônicas que possuem baixo pH, devido a presença de ácido cítrico. O consumo dessas bebidas durante a prática esportiva também contribui com refluxo gastroesofágico, sendo um fator de risco para a biocorrosão (Leite, 2019; Peters et al., 2000).

A prevalência de erosão dental em nadadores é alta, devido o contato dos dentes com a água da piscina quando possui pH ácido, e também pela frequência que ficam expostos durante as práticas esportivas. A utilização de substâncias, como o cloro gasoso, usado para evitar a proliferação de bactérias e algas na piscina, promove a redução do pH da água, devido a 
formação de ácido clorídrico no meio aquoso (Lussi \& Jaeggi, 2006). O que pôde ser verificado em um estudo que avaliaram 747 membros de um clube que utilizavam piscina tratada com gás cloro. Destes, 3\% dos não nadadores (9/295), 12\% dos nadadores não membros da equipe de natação (46/393) e 39\% dos membros da equipe de natação (23/59) apresentavam erosão dental. A água da piscina tinha pH 2,7, uma concentração de ácido aproximadamente 100.000 vezes a recomendada para piscinas (Centerwall et al., 1986). Durante a prática na piscina é recomendado que o pH esteja entre 7,2 a 8,0, porém muitos não seguem essas recomendações (Buczkowska-Radlińska et al., 2013).

A prevalência de erosão dental foi verificada em um grupo de nadadores da Polônia, os quais treinavam em piscina que a água era tratada com gás cloro. Foram avaliados três grupos de nadadores: 24 competidores juniores que apresentavam uma média de treino de 7 anos e com mais de 19 horas na piscina por semana, 38 competidores sêniores com média de treino por 10 anos com mais de 19 horas na piscina por semana, e um grupo de 69 nadadores recreativos, que nadavam uma ou duas vezes na semana, não passando de 2 horas por semana na piscina. A erosão dentária foi encontrada em mais de $26 \%$ dos nadadores competitivos e $10 \%$ dos nadadores recreativos. Embora o pH da água da piscina fosse neutro, era subsaturado em relação à hidroxiapatita. Concluíram que a duração e a quantidade de treino estão diretamente relacionadas ao risco de erosão dental. E que um risco aumentado de erosão pode estar relacionado à subsaturação da água da piscina com componentes de hidroxiapatita, como íons cálcio e fosfato (Buczkowska-Radlińska et al., 2013).

O tratamento da água das piscinas deve ser fiscalizado rotineiramente e deve ser dado preferência para a utilização de outras substâncias que não tenham potencial erosivo, como o hipoclorito de sódio, que possui pH alcalino (Dawes, 2003).

\subsubsection{Traumatismos}

As lesões bucais e faciais podem ocorrer nos tecidos moles, nos dentes e na sua estrutura de suporte, afetando o indivíduo fisicamente, funcionalmente e emocionalmente (Leci, 2020).

Os traumas esportivos representam de 14 a 39\% dos casos dos traumas dentários, e corresponde ao terceiro tratamento do trauma facial. É observado frequentemente o impacto entre os jogadores, durante quedas ou com objetos como bola e chuteiras. O futebol pode ser responsável por $50 \%$ dos traumas orofaciais devido sua alta popularidade entre amadores e profissionais (Leci, 2020).

Correa et al. (2010), avaliaram através de questionários, o trauma ocorrido em jogadores de futebol das 40 equipes da primeira e segunda divisões, da Liga Brasileira de Futebol de 2007. Das trinta e oito equipes que participaram da pesquisa, $71,1 \%$ relataram ocorrência de algum tipo de lesão dentária durante a prática do futebol, sendo as fraturas dentárias (74,1\%) e avulsões $(59,3 \%)$ as mais prevalentes. Apenas $21,6 \%$ dos profissionais recomendavam o uso de protetores bucais pelos jogadores, e quando a pesquisa foi realizada, quase a metade dos departamentos médicos não tinham dentistas no quadro de profissionais de saúde.

O trauma nos esportes é um problema comum, o qual pode ser minimizado e até mesmo prevenido. Torna-se importante o uso de protetores bucais e faciais para reduzir danos ao atleta, seja pelos ferimentos ou afastamento das atividades, o que pode prejudicar também a equipe, além de causar prejuízo financeiro para o clube. O risco de trauma pode ser reduzido em até 80\% com o uso destes dispositivos (Barberini et al., 2002; Bastida et al., 2010; Fraga et al., 2003).

Os protetores bucais afastam os tecidos moles dos dentes e funcionam como almofadas distribuindo as forças durante o trauma, podem ser de estoque ou universal, pré-fabricados e feitos sob encomenda. Os de estoque são encontrados em tamanho padrão, e são encontrados em lojas de artigos esportivos, possuem má adaptação e pouca retenção, dificultando a fala e respiração. Os pré-fabricados consistem em uma moldeira externa autopolimerizável ou moldeira termoplástica pré-formada que é plastificada em água quente e então moldada na boca pelo usuário. Possui uma razoável adaptação e melhor retenção quando comparado aos protetores de estoque. Os protetores bucais feitos sob medida, são confeccionados pelo cirurgião- 
dentista. Possuem melhor adaptação, retenção e distribuem melhor as forças de impacto (Barberini et al., 2002).

Os protetores bucais devem ser utilizados durante as práticas esportivas, principalmente naquelas que exista a chance de fortes impactos físicos, batidas com objetos ou quedas, como nas modalidades: Basquetebol, Futebol, Hóquei, Beisebol, Rugby, Ciclismo, Skate, Artes marciais, e em qualquer outra prática esportiva que traga risco a área bucal (Bastida et al., 2010).

Para evitar traumas é importante a utilização de protetores bucais e faciais, e seguir as orientações do cirurgiãodentista em relação a higienização e a substituição, principalmente em atletas em fase de crescimento para que não interfira no desenvolvimento da oclusão.

\subsubsection{Terceiros molares impactados}

A prática esportiva é mais comum em atletas jovens, época em que aparecem os primeiros problemas com os terceiros molares, conhecido popularmente como dente do siso. Devido à falta de espaço ou mal posicionamento, esses dentes podem ficar inclusos ou parcialmente erupcionados na cavidade oral. $\mathrm{O}$ mal posicionamento associado ao fato de erupcionarem em áreas de difícil higienização, pode aumentar o risco de cáries, infecções bucais, causando gengivite, hiperemia, dor, trismo, edema e halitose, comprometendo a saúde geral e rendimento (Costa, 2009; Martins, 2020).

A pericoronarite é a inflamação do tecido mole ao redor de um dente parcialmente irrompido, de caráter infeccioso ou não, comumente relacionado aos terceiros molares inferiores. Está relacionada ao mal posicionamento do dente e a higienização deficiente. Causa edema e dor intensa que pode irradiar para a região do ouvido e cabeça (Carregal, 2018).

Existem relatos na literatura, de que os terceiros molares impactados podem contribuir para fraturas angulares na mandíbula, sendo de maior prevalência, as fraturas causadas durante a prática de esportes. Foi observado um maior percentual de fraturas em atletas jovens e do sexo masculino (Martins, 2020).

A extração profilática do terceiro molar é defendida por alguns autores, no intuito de evitar fraturas no ângulo mandibular (Martins, 2020). Os atletas devem ser orientados para realizar avaliação clínica e radiográfica com dentista, para que se necessário, seja realizado tratamento.

\subsection{Odontologia e doping no esporte}

Em muitos tratamentos odontológicos é necessário associar o uso de medicamentos. Na Odontologia são utilizados diversos medicamentos como anti-inflamatórios esteroidais e não-esteroidais, antibióticos, analgésicos, anestésicos entre outros (Costa et al., 2015). Um cuidado especial é requerido ao tratar atletas, pois existem medicamentos proibidos no mundo esportivo devido ao doping.

O doping é conhecido em todo o mundo, e ocorre no meio esportivo quando o atleta, principalmente de elite, usa substâncias que contribuam para melhoria de desempenho (Fernandes, 2020). O cirurgião dentista deve prescrever medicamentos de forma correta para que não corra o risco de um resultado positivo no controle de doping, o que pode prejudicar o atleta em uma competição (Fernandes, 2020; Martins et al., 2013).

Anualmente é divulgada uma lista com as substâncias proibidas, que promovem maior desempenho, mas ferem o espírito esportivo e oferece risco para a saúde do atleta. Caso haja a necessidade da prescrição de um medicamento contendo uma substância restrita em sua composição, o Comitê Olímpico Brasileiro ou a respectiva Confederação deve ser contatado (Fernandes, 2020).

A Jamaicana Shelly-Ann Fraser foi acusada em um exame antidoping, por utilizar oxicodona, um analgésico opioide utilizado para dores moderadas a intensas. Mesmo alegando que a substância havia sido utilizada para tratar uma dor de dente, a Associação Internacional das Federações de Atletismo suspendeu a campeã por seis meses das competições (Padilha et al., 
2016).

O cirurgião-dentista deve estar ciente das normas que o atleta está sujeito. Deve conhecer o uso de medicamentos restritos e seus efeitos, pois um resultado positivo no controle de doping pode trazer consequências irreversíveis para a vida do atleta (Fernandes, 2020).

\section{Considerações Finais}

Pôde-se verificar a alta prevalência de doenças bucais em atletas, e a importância da participação do cirurgião-dentista nas equipes multiprofissionais, para promoção da saúde bucal. As doenças bucais que mais acometem os atletas são: cárie dentária, doença periodontal, má oclusão, DTM, erosão dental, traumatismos orofaciais, terceiros molares inclusos.

Os atletas, amadores ou profissionais, devem estar cientes da influência da saúde bucal, na saúde geral, qualidade de vida e rendimento físico. Pois, as doenças bucais podem comprometer a eficiência mastigatória, favorecer traumas dentários, gerar complicações cardiovasculares e respiratórias, interferir na hipertrofia e no reparo muscular, além do fato da dor de dente diminuir a disposição e capacidade de concentração do atleta.

É importante a implementação de estratégias preventivas, para orientação sobre higiene bucal e informação sobre os hábitos bucais deletérios. As orientações englobam cuidados na dieta, pois alimentos ácidos e ricos em carboidratos, favorecem o desenvolvimento da erosão dental e da cárie dentária. As consequências do bruxismo, que pode ocasionar dores e desgaste dental, e a respiração bucal, que influencia no cansaço e na qualidade de sono. Deve-se enfatizar também a importância da utilização de protetores bucais e faciais, para evitar traumatismos e reduzir danos ao atleta.

O cirurgião-dentista, que se dedica a Odontologia do Esporte, deve ter cuidado na seleção de medicamentos ao tratar as doenças bucais, pois alguns medicamentos podem acusar resultado positivo no controle de doping, gerando consequências irreversíveis para o atleta.

Recomenda-se que todos os atletas, independentes da modalidade esportiva, tenha acompanhamento de um especialista da área odontológica, para tratamento e manutenção da saúde bucal, pois a redução do desempenho devido ao comprometimento da saúde bucal é inaceitável e evitável.

\section{Referências}

Abou-Raya, S., Naeem, A., Kheir, H. A.-E., \& El Beltagy, S. (2002). Coronary artery disease and periodontal disease: is there a link? J Angiology, 53(2), 141148. https://doi: 10.1177/000331970205300203.

Alves, D. C. B., Anjos, V. D. L. d., Giovannini, J. F. B. G., Lima, R. P. E., \& Mendonça, S. M. S. (2017). Odontologia no esporte: conhecimento e hábitos de atletas do futebol e basquetebol sobre saúde bucal. J Revista Brasileira de Medicina do Esporte, 23(5), 407-411. http://dx.doi.org/10.1590/1517869220172305170315 .

Amorim, R. F., \& Añez, I. N. F. (2017). Importância da odontologia esportiva no condicionamento do atleta. [Trabalho de Conclusão de Curso, Centro Universitário São Lucas]. http://hdl.handle.net/123456789/2010.

Andrade, L. G. N., da Silva, M. A., Leite, J. J. G., \& de Castro Filho, C. S. (2017). Os desafios da odontologia no esporte: uma nova perspectiva: revisão de literatura. J Revista Diálogos Acadêmicos, 6(2), 92-98. http://revista.fametro.com.br/index.php/RDA/article/view/145.

Antunez, M. E. M., \& dos Reis, Y. B. (2010). O binômio esporte-odontologia. J Adolescência e Saúde, 7(1), 37-39.

Ashley, P., Di Iorio, A., Cole, E., Tanday, A., \& Needleman, I. (2015). Oral health of elite athletes and association with performance: a systematic review. $J$ British Journal of Sports Medicine, 49(1), 14-19. https://doi:10.1136/bjsports-2014-093617

Assis, C. (2013). Os rumos da odontologia do esporte no Brasil. J Revista Brasileira de Odontologia, 70(2), 160-164. http://revodonto.bvsalud.org/scielo.php?script=sci_arttext\&pid=S0034-72722013000200013.

Barberini, A. F., Aun, C. E., \& Caldeira, C. L. (2002). Incidência de injúrias orofaciais e utilização de protetores bucais em diversos esportes de contato. J Rev. Odontol. UNICID, 14(1), 7-14. http://www.odontologiasobral.ufc.br/wp-content/uploads/2009/12/protetores.pdf.

Bastida, E. M., Peron, R. A. F., Queiroz, A. F., Hayacibara, M. F., \& Terada, R. S. S. (2010). Prevalência do uso de protetores bucais em praticantes de artes marciais de um município do Paraná. J Revista Brasileira de Odontologia, 67(2), 194-198. http://revista.aborj.org.br/index.php/rbo/article/view/192. 
Bastos, R. d. S., Vieira, E. M. M., Simões, C. A. D., Sales Peres, S. H. d. C., Caldana, M. d. L., Lauris, J. R. P., \& Bastos, J. R. d. M. (2013). Odontologia desportiva: proposta de um protocolo de atenção à saúde bucal do atleta. Revista Gaúcha de Odontologia, 61, 461-468. http://revodonto.bvsalud.org/scielo.php?pid=S1981-86372013000500006\&script=sci_arttext.

Batista, T. R. d. M., Vasconcelos, M. G., \& Vasconcelos, R. G. (2020). Fisiopatologia da cárie dentária: entendendo o processo carioso. J Rev. Salusvita, 169187. https://secure.unisagrado.edu.br/static/biblioteca/salusvita/salusvita_v39_n1_2020/salusvita_v39_n1_2020_art_14.pdf.

Bonecker, M., Guedes-Pinto, A. C., \& Fernandes, F. (2012). Cárie dentária. In: Guedes-Pinto, A.C. Odontopediatria. (8a ed.), Santos, $313-346$.

Bonotto, D. (2013). Prevalência de disfunção temporomandibular em atletas de esportes de contato. [Tese de Doutorado, Pontifícia Universidade Católica do Paraná]. http://cev.org.br/arquivo/biblioteca/4030376.pdf.

Bonotto, D., \& Bonotto, D. M. V. (2016). Disfunção temporomandibular em atletas. In: Namba, E. L., Padilha, C. Odontologia do Esporte: Um novo caminho. Uma nova especialidade. Florianópolis: Editora Ponto, 32-53.

Buczkowska-Radlińska, J., Łagocka, R., Kaczmarek, W., Górski, M., \& Nowicka, A. (2013). Prevalence of dental erosion in adolescent competitive swimmers exposed to gas-chlorinated swimming pool water. J Clinical oral investigations, 17(2), 579-583. http://doi: 10.1007/s00784-012-0720-6. Epub 2012 Apr 3.

Carregal, M. G. (2018). Pericoronarite: etiologia, epidemiologia, microbiota, tratamento e complicações. [Monografia Especialização em Cirurgia e Traumatologia Buco Maxilo-Facial com Práticas Hospitalares Avançadas, Universidade Federal de Minas Gerais, Belo Horizonte]. https://repositorio.ufmg.br/bitstream/1843/ODON-B8EMN3/1/monografia__mateus_corradi_carregal.pdf.

Centerwall, B. S., Armstrong, C. W., Funkhouser, L. S., \& Elzay, R. P. (1986). Erosion of dental enamel among competitive swimmers at a gas-chlorinated swimming pool. J American Journal of Epidemiology, 123(4), 641-647. https://doi: 10.1093/oxfordjournals.aje.a114283.

CFO, C. F. d. O. (Acesso em: 24 de jul. 2020). CFO promove a III Assembleia Nacional de Especialidades Odontológicas - ANEO http://cfo.org.br/destaques/cfo-promove-a-iiiassembleia-nacional-de-especialidades-odontologicas-aneo

Correa, M. B., Schuch, H. S., Collares, K., Hallal, P. C., \& Demarco, F. F. (2010). Survey on the occurrence of dental trauma and preventive strategies among Brazilian professional soccer players. J Journal of applied oral science, 18(6), 572-576. http://doi: 10.1590/s1678-77572010000600007.

Costa, S., Alencar, D., Maia, C., Costa, I., \& Fidelis, R. (2015). Odontologia desportiva: melhor performance com a atuação do dentista na prática da atividade física. J Col Pesq Educ Física, 14, 131-138. https://fontouraeditora.com.br/periodico/upload/artigo/1215_1505234625.pdf.

Costa, S. d. S. (2009). Odontologia desportiva na luta pelo reconhecimento. J Revista de Odontologia da Universidade Cidade de São Paulo, 21(2), 162-168. http://arquivos.cruzeirodosuleducacional.edu.br/principal/old/revista_odontologia/pdf/maio_agosto_2009/Unicid_2(2)_2009_162-168.pdf.

Cury, J. A., Tenura, L. M. A., \& Tabchoury, C. T. P. (2017). Bioquímica Oral.

Dalri, C. (2020). The influence of poor oral health on muscle injuries. In: Padilha C. et al. Sports Dentistry - Written by women, Balneário Camboriú, 71-73.

Dawes, C. (2003). What is the critical pH and why does a tooth dissolve in acid? J Journal-Canadian Dental Association, 69(11), 722-725. http://www.cdaadc.ca/jcda/vol-69/issue-11/722.html.

Fernandes, F. (2020). Dentist and Doping in sports. In: Padilha C. et al. Sports Dentistry - Written by women, Balneário Camboriú, p. $96-98$.

Flake, N. M., Hermanstyne, T. O., \& Gold, M. S. (2006). Testosterone and estrogen have opposing actions on inflammation-induced plasma extravasation in the rat temporomandibular joint. J American Journal of Physiology-Regulatory, Integrative Comparative Physiology, 291(2), R343-R348. http://doi: 10.1152/ajpregu.00835.2005.

Fraga, A. C. d. A., Teófilo, L. T., \& Alencar, A. H. G. d. (2003). Protetores bucais: regulamentação do uso, tipos, conscientização, eficiência e confecção. J Arq. odontol, 297-310. https://pesquisa.bvsalud.org/portal/resource/pt/lil-415677.

Freitas, M. R. (2013). Desenvolvimento da oclusão. In: Janson G. et al., Introdução à Ortodontia, Série Abeno, p. 34-61.

Frese, C., Frese, F., Kuhlmann, S., Saure, D., Reljic, D., Staehle, H., \& Wolff, D. (2015). Effect of endurance training on dental erosion, caries, and saliva. J Scandinavian journal of medicine science in sports, 25(3), e319-e326. https://doi: 10.1111/sms.12266. Epub 2014 Jun 11.

Fronza, H. (2020). Periodontal diseases in athletes: is there a relation with systemic health? In: Padilha C. et al. Sports Dentistry - Written by women, Balneário Camboriú, p. 75-79.

Garib, D. G., Janson, G., C.C., M., \& Sanches, F. S. H. (2013). Etiologia das maloclusões. In: Janson et al., Introdução à Ortodontia, Série Abeno, p. 62-75.

Gay-Escoda, C., Pereira, D. M. V. D., Ardèvol, J., Pruna, R., Fernandez, J., \& Valmaseda Castellón, E. (2011). Study of the effect of oral health on physical condition of professional soccer players of the Football Club Barcelona. J Medicina Oral, Patología Oral y Cirugia Bucal, 16(3), 436-439. https://doi: $10.4317 /$ medoral. $16 \mathrm{e} 436$

Gialain, I. O. (2015). Estudo da eficácia de protetores bucais para esporte quanto à espessura por meio de análise em elementos finitos Universidade de São Paulo]. https://www.teses.usp.br/teses/disponiveis/23/23152/tde-19012016-162429/en.php.

Gil, A. C. J. C. e. p. d. p. E. S. P. A. (2010). Como elaborar projetos de pesquisa/-12. Atlas, 2009.

Grippo, J. O., Simring, M., \& Coleman, T. A. (2012). Abfraction, abrasion, biocorrosion, and the enigma of noncarious cervical lesions: A 20-year perspective. J Journal of Esthetic Restorative Dentistry 24(1), 10-23. https://doi: 10.1111/j.1708-8240.2011.00487.x

Jacobs, W. H. (1930). Boxer's appliance. J Dental Digest, 36, 201. 
Leci, L. (2020). Orofacial trauma in soccer players. In: Padilha C. et al. Sports Dentistry - Written by women, Balneário Camboriú, p. 46-50.

Leite, V. B. C. (2019). Prevalência de erosão dental em atletas de esportes de resistência [Trabalho Conclusão de Curso (Graduação em Odontologia), Centro de Ciências da Saúde, Universidade Federal de Santa Catarina, Florianópolis]. https://repositorio.ufsc.br/handle/123456789/201613.

Lima, A. C. A., Cardoso, E. L. d. O., Ferreira Junior, P. R. L., Bento, G., \& Haddad, M. F. (2019). Odontologia do esporte: revisão de literatura. J Archives of Health Investigation, 8(12), 836-845. https://doi.org/10.21270/archi.v8i12.4646.

Lussi, A., \& Jaeggi, T. (2006). Occupation and sports. In: Dental Erosion (Vol. 20, 106-111). Karger Publishers.

Lussi, A., \& Jäggi, T. (2008). Erosion—diagnosis and risk factors. J Clinical oral investigations, 12(1), 5-13. https://doi: 10.1007/s00784-007-0179-z.

Martins, C. (2020). Mandibular fractures associates with third molar impaction. In: Padilha C. et al. Sports Dentistry - Written by women, Balneário Cambori, $51-54$.

Martins, M., Silva, T., Viana, F., Vasconcelos Filho, J., Ramalho Filho, A., \& Lima, D. (2013). Medicamentos de uso odontológico considerados proibidos pela Agência Mundial Anti-Doping (WADA). J Coleção Pesquisa em Educação Física, 12(2), 95-102.

Nakajima, T., Honda, T., Domon, H., Okui, T., Kajita, K., Ito, H., Takahashi, N., Maekawa, T., Tabeta, K., \& Yamazaki, K. (2010). Periodontitis-associated up-regulation of systemic inflammatory mediator level may increase the risk of coronary heart disease. Journal of Periodontal Research, 45(1), 116-122. https://doi: 10.1111/j.1600-0765.2009.01209.x.

Namba, E. L. (2018). Protetor Bucal Esportivo “Novos Conceitos”. Retrieved 30 de Setembro from https://www.sportsdental.com.br/protetor-bucal-esportivonovos-conceitos

Nazir, M. A. (2017). Prevalence of periodontal disease, its association with systemic diseases and prevention. J International Journal of Health Sciences, 11(2), 72-80. https://www.ncbi.nlm.nih.gov/pmc/articles/PMC5426403.

Needleman, I., Ashley, P., Fine, P., Haddad, F., Loosemore, M., de Medici, A., Donos, N., Newton, T., van Someren, K., \& Moazzez, R. (2015). Oral health and elite sport performance. J British Journal of Sports Medicine, 49(1), 3-6. https://doi: 10.1136/bjsports-2014-093804.

Needleman, I., Ashley, P., Petrie, A., Fortune, F., Turner, W., Jones, J., Niggli, J., Engebretsen, L., Budgett, R., \& Donos, N. (2013). Oral health and impact on performance of athletes participating in the London 2012 Olympic Games: a cross-sectional study. J British Journal of Sports Medicine, 47(16), 1054-1058. https:// doi: 10.1136/bjsports-2013-092891. Epub 2013 Sep 24.

Nunes, A. F., Fogaça, C. L., Grande, R. S., Kayser, E. G., \& de Souza, B. C. (2020). Comparação de técnicas ortodônticas e uma sugestão de tratamento para o paciente atleta. J Disciplinarum Scientia Saúde, 21(1), 267-283. https://periodicos.ufn.edu.br/index.php/disciplinarumS/article/view/3271.

Okeson, J. P., \& de Leeuw, R. (2011). Differential diagnosis of temporomandibular disorders and other orofacial pain disorders. J Dental Clinics, 55(1), 105120.

Padilha, A. C. L. (2015). O trabalho do cirurgião-dentista no contexto esportivo: conhecendo as bases para um trabalho interdisciplinar. [Dissertação (Mestrado) - Programa de Pós-Graduação em Odontologia, Universidade Federal de Santa Catarina, Florianópolis].

Padilha, C., \& Namba, E. L. (2016). Introdução A Odontologia do Esporte. In: Namba, E. L., Padilha, C. Odontologia do Esporte: Um novo caminho. Uma nova especialidade. p. 32-53. Editora Ponto.

Padilha, C., Ribas, C. R., \& Namba, E. L. (2016). Doping e a Odontologia do Esporte. In: Namba, E. L., Padilha, C. Odontologia do Esporte: Um novo caminho. Uma nova especialidade. p. 266-272. Editora Ponto.

Pastore, G. U., Moreira, M., Bastos, R., Galotti, M., \& Leonardi, M. F. d. P. (2017). Odontologia do esporte-uma proposta inovadora. J Revista Brasileira de Medicina do Esporte, 23(2), 147-151. https://www.scielo.br/scielo.php?pid=S1517-86922017000200147\&script=sci_abstract\&tlng=pt.

Peters, H., Wiersma, J., Koerselman, J., Akkermans, L., Bol, E., Mosterd, W., \& De Vries, W. (2000). The effect of a sports drink on gastroesophageal reflux during a run-bike-run test. J International Journal of Sports Medicine, 21(01), 65-70. https://doi: 10.1055/s-2000-8858.

Raquel, G., Namba, E. L., Bonotto, D., Rosa, E. A. R., Trevilatto, P. C., Machado, M. Â. N., Vianna-Lara, M. S., \& Azevedo-Alanis, L. R. (2016). The use of a custom-made mouthguard stabilizes the electromyographic activity of the masticatory muscles among Karate-Dō athletes. J Journal of bodywork movement therapies, 21(1), 109-116. https://doi: 10.1016/j.jbmt.2016.05.007.

Rodrigues, C. K. e. a. (2006). Bruxismo: uma revisão da literatura. Publicatio UEPG: Ciências Biológicas e da Saúde, 12(3), 617-643.

Rother, E. T. J. A. p. d. e. (2007). Revisão sistemática X revisão narrativa. Acta paul. enferm, 20(2), v-vi. https://www.scielo.br/scielo.php?script=sci_arttext\&pid=S0103-21002007000200001.

Saga, A. Y., Maruo, I. T., Maruo, H., Guariza Filho, O., Camargo, E. S., \& Tanaka, O. M. (2011). Treatment of an adult with several missing teeth and atrophic old mandibular first molar extraction sites. J American journal of Orthodontics Dentofacial Orthopedics, 140(6), 869-878. https://doi.org/10.1016/j.ajodo.2010.06.027.

Santos-Coluchi, G. G., \& Simplício, H. (2011). Classificação das Más Oclusões. In: Ortodontia e Fonoaudiologia na Prática Clínica (p. 81-107). Revinter.

Silveira, V. R. S., \& Alves, A. P. N. N. (2009). Perfil celular e mediadores químicos na Doença Periodontal associada ao biofilme dental-Revisão de literatura. Periodontia, 73-79. http://www.interativamix.com.br/SOBRAPE/arquivos/set_2009/artigo10.pdf.

Soares, I. S. Q., Miranda, A. F. V., Assencio-Ferreira, V., \& Di Ninno, C. (2004). Bruxismo: desempenho da mastigação em adultos jovens. J Rev CEFAC, 6(4), 358-362. 
Research, Society and Development, v. 10, n. 3, e51510313683, 2021

(CC BY 4.0) | ISSN 2525-3409 | DOI: http://dx.doi.org/10.33448/rsd-v10i3.13683

Souza, B. C. (2013). Impacto da condição periodontal nos níveis séricos de marcadores inflamatórios e no processo de reparo muscular de ratos wistar treinados e sedentários. [Dissertação de Mestrado, Universidade Federal do Rio Grande do Sul, Porto Alegre]. http://hdl.handle.net/10183/79060

Souza, B. C. (2017). Saúde bucal do atleta: Uma relação paradoxal com a qualidade de vida. J Brazilian Journal of Surgery Clinical Research, 20(1), 147-150. https://www.mastereditora.com.br/periodico/20170905_173145.pdf.

Souza, B. C., \& Ribas, M. E. (2009). Associação entre condição periodontal e níveis séricos de creatina quinase em jovens atletas jogadores de futebol. [Trabalho de Conclusão de Curso (Graduação em Odontologia), Universidade Federal do Rio Grande do Sul, Porto Alegre]. http://hdl.handle.net/10183/18459.

Souza, B. C., Teixeira, B. C., \& Ribas, M. E. (2016). Doenças infectoinflamatórias bucais e alterações no tecido muscular. In: Namba, E. L., Padilha, C. Odontologia do Esporte: Um novo caminho. Uma nova especialidade. (p. 72-83)

Souza, L. A., Elmadjian, T. R., Dias, R. B., \& Coto, N. P. (2011). Prevalence of malocclusions in the 13-20-year-old categories of football athletes. J Brazilian oral research, 25(1), 19-22. https://www.scielo.br/scielo.php?script=sci_arttext\&pid=S1806-83242011000100004.

Trigo, M. (2002). O Eterno Futebol. Brasília, Editora Thesaurus.

Weiler, R. M. E., de Souza Vitalle, M. S., Mori, M., Kulik, M. A., Ide, L., Pardini, S. R. d. S. V., \& Santos, F. M. (2010). Prevalence of signs and symptoms of temporomandibular dysfunction in male adolescent athletes and non-athletes. J International Journal of Pediatric Otorhinolaryngology, 74(8), 896-900. https:/ doi: 10.1016/j.ijporl.2010.05.007.

Werutski, L., \& Falchetti, G. (2016). Etiologia da maloclusão. In: Namba, E. L., Padilha, C. Odontologia do Esporte: Um novo caminho. Uma nova especialidade. (p. 198-207). Editora Ponto. 